Clin Pediatr (Phila) Vol. 12(1):35-41 (1973)

ISSN: (paper 0009-9228)(online 1938-2707)

doi:10.1177/000992287301200110

This is a peer reviewed pre-print version of the following article: The Art of Medicating Hyperkinetic Children: A Number of Practical Suggestions, which has been published in final form at:

http://www.sagepub.com

http://cpj.sagepub.com

http://cpj.sagepub.com/content/12/1/35.full.pdf+html

(C) 1973 Sage Publications, Inc.

\title{
The Art of Medicating Hyperkinetic Children: A Number of Practical Suggestions
}

\section{Eugene Arnold, M.D., Assistant Professor, Psychiatry and Pediatrics, Ohio State University, Columbus, Ohio 43210.}

\begin{abstract}
The psychologic aspects of the prescribing, giving, and taking of medicines are especially important when trying to modify the emotion-laden behavior of hyperkinetic children. The physician should start by cultivating positive expectations in child, parents, and teacher. This is necessary in order to break up existing complex vicious cycles of negative expectations, low self-esteem, discouragement, resentment, misbehavior, and poor achievement. One promotes the child's cooperation by building rapport from the first contact and by negotiating a medication contract directly with him. The child needs to know not only that his past misbehavior or failures are not being held against him but also that better is expected of him now that he has medical help. Parents deserve unhurried clarification of questions and should be warned about side effects. The time taken to talk directly with the teacher is most valuable. Direct communication can help set up positive expectations in school and elicit objective information about the child's day-to-day performance.
\end{abstract}

The literature has recently produced an abundant harvest of articles describing the diagnosis and treatment of the hyperkinetic handicap in children with minimal brain dysfunction. Many of these articles emphasize the importance of the psychologic dimension in managing such children, ${ }^{1,2}$ with recommendations for counseling, parent guidance, etc. However, they direct relatively little attention to the psychologic aspects of medicine-prescribing, medicine-giving, and medicine-taking. Nevertheless, these often emotion-charged procedures vitally affect the efficacy of drugs which are being given to modify behavior.

This article will elucidate some of the psychologic dimensions of medicating, and suggest ways of utilizing this knowledge to promote the efficacy of individual case treatment.

\section{Choosing the Drug}

Drug efficacies, discussed by many authors, ${ }^{3-5}$ are not the focus of this article. However, it is pertinent to point out the preeminence of stimulants in the treatment of hyperkinetic children because of their dramatic benefit and relative safety for the children in whom they are efficacious, $^{4,6}$

Unfortunately, their efficacy is not uniform. This may lead a busy clinician to choose a more predictable drug, such as a tranquilizer. Though the tranquilizers may more uniformly affect the whole spectrum of hyperkinetic children, they do not seem as beneficial as stimulants with a large proportion of such children. Furthermore, some of them carry risks, such as allergic reactions, blood dyscrasia, and organ toxicity, which the stimulants do not. Therefore, in light of 
current knowledge, it seems worthwhile for the clinician to try a stimulant first, keeping the tranquilizers and other drugs in reserve if the child does not benefit from it. The balance of this article will assume that such a course has been chosen.

One disadvantage of stimulants is the potential for addictive abuse. Though such addiction has never been reported in a hyperkinetic child, sometimes the child's relatives or friends may abuse his supply of stimulants. In such situations, when there is no other means of control, another type of medication may be advisable. Even when a danger of drug abuse by relatives does not obtain, concern about giving a child a medication listed by the federal government as a "dangerous drug" often persists. The physician needs to deal with this concern, preferably in a prophylactic way.

\section{Setting a Rational Tone}

Implicit in this report is the assumption that the physician has already established rapport with the child and his parents by means of warmth, empathy, patience, respect, and a willingness to listen exhibited during the initial contact, the history taking, and the examination (or during previous pediatric care). Because of the trust generated by such rapport, they will probably accept the physician's frank explanation of the medication being prescribed. In fact, nondefensive frankness will confirm the patient's (and his parents') confidence that they can trust the doctor and his treatment. An opposite course - not labeling or explaining what kind of medicine is being given-runs the risk of undermining the trust which the patient and his family must have in the physician in order for him to help them.

One brief way of informing the child and his parents about the medication is to name the drug and ask them if they have heard of it. If they have, stop and let them describe their understanding of it. If they seem ignorant of it, remark that they may have read or heard about it for two reasons: 1) it is one of many drugs which have been abused by adults to the point of addiction, and 2) it has an unexpected calming effect on hyperactive children, for whom no cases of addiction have ever been reported. If they seem genuinely satisfied with this explanation, it does not need to be pursued. However, if they wish to talk further about it, or ask questions, or look worried or anxious, questions should be asked, while allowing them to ventilate their concerns. All answers should be given in a frank, open, nondefensive way.

It is not necessary for the physician to "sell" the parents on the value or safety of the medication, but he does need to provide impartial information, advice, and recommendations. He is the expert whom they trust. The final agreement about treatment of the child, of course, rests with his parents or other legal guardians. The physician, by his calm, reasonable disclosure of pertinent facts helps the parents (and the patient) accept the medication in a rational way.

\section{Parlaying Placebo}

The physician should not discourage one particular less-than-rational accompaniment of medication. In fact, he should cultivate it for the benefit of his patients. The self-fulfilling prophecy of expected cure, most clearly manifested and measurable in "the placebo effect," has been the healer's (and the patient's) friend for centuries. ${ }^{7}$ It has accounted in large part for the apparent efficacy of "eye of toad and tongue of newt" remedies. Because it complicates scientific evaluation of drugs and because it has been exploited by witch doctors, faith healers, shamans, and charlatans as their sole therapeutic offering, placebo effect has developed a shady reputation among modern physicians. To scorn this valuable therapeutic tool would be shortsighted, however. The compleat physician should cultivate it to give his patients the benefit both of this "wisdom of the ages" and of more scientific therapeutics. 
This approach seems especially true for the hyperkinetic patient. Many of his problems are secondary psychosocial elaborations of the original neurophysiologic deficit. Figure 1

incorporates some of the possible complex vicious cycles that can result. Note that once these are established, the minimal brain dysfunction itself can be stricken from the picture (as by effective medication) and the vicious cycle may continue unabated, running on the momentum from its feedback mechanisms. Many of those feedback mechanisms involve expectations, either the child's expectations for himself or others' expectations (parents, teachers, peers). One way of attacking these depressive expectations is to substitute expectations of change for the better, with the hope of establishing a "virtuous circle" via the constructive self-fulfilling prophecy of placebo effect. For some children this effect of medication may be of more benefit than the pharmacologic effect.

Enhancing the placebo influence of the unpredictable stimulants without painting one's self into a corner requires thought and planning until sufficient practice has made it second nature. One method begins by stating that there are many different medicines which are helpful to the kind of problem the child has, that one of these medicines may be better for one child and another better for another child, and that there is no sure way of telling ahead of time which is best for a given child. Furthermore, the dose required varies from child to child. Therefore, no one should get discouraged if the dosage or even the type of medicine has to be changed several times in order to find the best dose of the best medicine for this particular child.

This sort of introduction allows for the possibility that the first medicine tried may not be satisfactory, while giving optimistic assurance that another medicine may be better. Most people, on hearing this, will tend to pick up the optimistic, confident note and forget the implication of uncertainty. However, in the event that the first medication should prove unsatisfactory, the bets have been hedged. At that time, the patient and his parents can be reminded hy the physician that it might be necessary to change medicine.

After having built in these necessary disclaimers, the physician should explain in more detail the expected benefits: the child will probably find it easier to concentrate, to control himself (including his temper), to ignore distractions, and to do what he wants to do when he wants to do it. Medication will not make him be good, but will make it possible for him to behave in the way in which he has always wanted to, but had difficulty doing until the present. Parents should expect to find that he learns better from experience, is easier to train, and is more even-tempered. They may find that where previously they had to whack him to get his attention, he may now break into tears if they raise their voices at him, requiring a more gentle approach. They should also expect him temporarily to lose his appetite, possibly become whiny and depressed, and perhaps have trouble sleeping. Coupling the mention of side effects with the description of anticipated benefits tends to make them more acceptable. It also tends to make them reinforce the positive expectations. When they occur as predicted, they confirm the credibility of the doctor who also predicted the benefits: "The medicine is working just like the doctor said it would."

Teachers and other school personnel need to be included in the placebo effort, but this will be dealt with in more detail in the section on school contact.

\section{Art Over Science}

Those who may have scientific scruples about thus muddying the therapeutic waters with a highly cultivated placebo effect should consider the following. Without a double-blind procedure and matched dosage forms, an unknown amount of placebo effect will creep in anyway, unless the physician deliberately creates the impression that the medicine is worthless, liven the latter may merely "change the sign" and result in a negative placebo effect (expectation of harm). This, of 
course, would be neither sound science nor good medical practice.

Distinguishing placebo cures after the fact does not really present much of a practical problem, anyhow. Doubtful cases can be taken off medication gradually after a few months (after a psychosocial virtuous circle has replaced the vicious one). Even though the physician mentioned at the beginning that the medicine may have to be taken for several years, people ate usually willing to believe that the problem has been cured and medication is no longer needed. In fact, it is not unusual for a patient (or his parents) to neglect medication against doctor's orders when the presenting complaint has abated. With the more sophisticated, the physician may wish to share his thought about the breakup of the vicious circle.

Needless to say, the withdrawal of medication should be announced in a confident but empirical manner such as "Things are going well enough now so that I think we can try stopping the medicine. It may very well be that you (he) no longer need(s) it." This allows for the possibility of restarting the medicine if the clinical picture deteriorates, without implying an expectation that this will happen. Most children (and their parents) welcome such a move, though an occasional child may have misgivings about stopping the medication. Such a child should be given a chance to ventilate his concerns and be assured that he will not be let down. For the child who has deep concern, tapering of the medicine in order to "wean" him would be desirable.

\section{The Child Is the Star of the Therapeutic Team}

The child's cooperation is essential to the success of the whole treatment program. Perhaps one of the most convincing arguments against the likelihood of such children becoming addicted is the number of them who refuse the medicine for a variety of reasons. With many, it is merely the taste of the medication. With some, it is a matter of principle: the child is not willing to admit that there is anything wrong with him for which he needs medication. With others, the medication becomes a new focus for a long-standing power struggle between the parents and child. With still others, it is more a matter of negligence and carelessness. The child's resistance to the taking of medicine is one of the most common causes of therapeutic failure. That is why the physician must cultivate the child's cooperation and try to negotiate a medication contract directly with him. This must be done with the knowledge and consent of the parents, of course, and usually can be done in their presence.

The groundwork must be laid in the first contact with the child. The old pediatric axiom that the child is the patient, not his parents, nowhere has greater utility than in this situation. The physician, by words and action, must convey to the child his genuine respect and consideration for him as a person. When the child and his parents are together in the same room, questions, explanations, and advice should be directed towards the child as much as possible. Most parents are pleased to see this kind of consideration, and are content to get most of their information by "listening in." They, of course, should also be given a chance to ask any questions about which they may wish additional clarification.

Occasionally, despite the physician's best efforts, the child may inexplicably be unwilling to consider the necessity of taking medication, even though his parents are willing for him to. In such a situation, the physician may be more firm. It is better for the physician than for the parents to persuade, in order to bypass the possibility of entangling the medication in a long-standing feud between the child and his parents. One useful ploy is to ask the sports-minded boy to take the medication "under protest," implying that he is not submitting or admitting any weakness in himself, but is merely being a good sport about it. Few boys can turn down such an appeal from a doctor who has been treating them with genuine respect and consideration. 


\section{The School's Part in Medication}

Direct contact should be made with someone at school, preferably the child's teacher. The school's cooperation is essential to the success of treatment for several reasons, and should not be left to chance or to the vagaries of a verbal message sent through the child or his parents.

Even before treatment, direct information from the teacher is helpful in making an accurate diagnosis. Of course, some judgment must be exercised in assessing such information. For example, the physician should be skeptical about the validity of a diagnosis from a teacher who can cite nine examples from his class to demonstrate the value of medication and is convinced that he has found another hyperkinetic child. On the other hand, a teacher who seems prejudiced against medication, has no other children in his class taking it, and only reluctantly admits that this child is not normal may be a hyperkinetic child even when he tries to hide the fact. Nevertheless, teachers remain the best source of objective information about a child because they observe the child many hours in various situations, have many normal children of the same age for comparison, and are not usually as overwhelmed by emotional involvement as parents tend to be.

For these reasons, regular reports from the teacher on the child's progress, either in general or with regard to specific target symptoms, constitute one of the best ways to monitor the efficacy of medication. This is especially true when the chief complaint is trouble in school with not much trouble at home: school is where the action is. Even when the problem involves the home, the teacher's observation of possible changes can be useful in managing the medication.

An important additional reason for contacting the school was mentioned in the discussion of placebo effect. Full exploitation of this valuable therapeutic tool must include the school. Usually many of the expectations maintaining the vicious circle in Figure 1 reside in the teacher and other people at school, including classmates. Hence the importance of contacting the teacher or a reasonable substitute to establish better expectations. This can usually be accomplished by being optimistic when asking for the periodic progress reports. For instance, the teacher can be told, "In order to adjust the dosage accurately, I would appreciate weekly reports from you on how much he has improved. The benefits I am particularly interested in are ..." The description of anticipated benefits should be given to the teacher just as it was to the child and his parents. For the teacher who is not already familiar with the concept of the hyperkinetic child, a short explanation will cultivate his cooperation and will help him to take a more effective approach to the child.

Occasionally, the teacher and parents may have been blaming each other for the child's problem, sometimes because of a fear of being blamed themselves. Efforts should be made to remove the problem from a blame-placing context. If some blame must be placed, it should be placed on the child's handicap and on the consequent frustrations to the child, his parents, and the teacher. In this way all concerned may feel like joining forces to fight the common enemy, the handicap. 


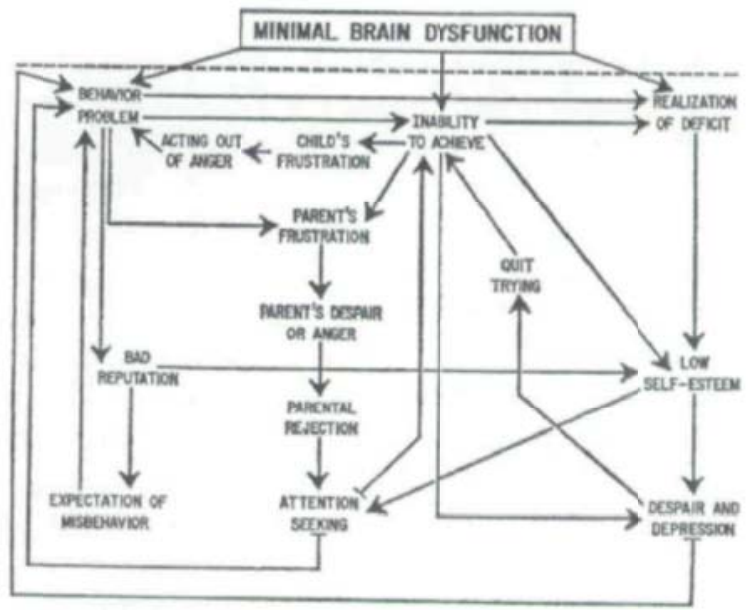

Fig. 1. Complex vicious cycle sometimes originated by minimal brain dysfunction. Can be maintained by own feedback even after original handicap adequately treated or outgrown (indicated by dotted line).

As mentioned, the five or ten minutes needed for school contact may be the most useful time spent for the benefit of the child. It is appropriate to charge the child's parents a reasonable fee for this time. In the interest of efficiency, subsequent routine reports from the teacher can be by note, telephone message, or by setting aside a time each day when such calls can be received.

\section{Followup}

Parents appreciate having a certain time when they know they can routinely reach the physician by telephone. This ordinarily is the most efficient means of monitoring medication during the initial weeks or months. The effects of medication should be reassessed at least weekly at the beginning, in order to adjust the dosage. It is not necessary to see the child each time; regular telephone contacts are adequate. Again, the physician should feel free to charge for this if it is not included in his initial fee. In the long run, he will be saving the parents money and providing better medical service.

Because of the wide variation in individual dosages, the starting dose is best made low. Increases may be made weekly (or more often) until either benefits or intolerable side effects are reached. The necessary monitoring information can be collected by telephone from parents or teacher, with some provision for the child to report how he feels.

Once the effective dose is established, the child need not be seen more than two or three times a year, as long as things are proceeding satisfactorily. About once a year (or more often, if desired) a trial withdrawal of medication can be attempted. A good way to do this is to stop it during summer vacation and let the child return to school without it in the fall, restarting it only if necessary. In fact, those who had trouble only in school can often get by from the beginning with taking stimulants only on school days.

\section{Tolerance}

The latter plan, besides being safer and cheaper, also helps prevent tolerance. Though tolerance to the "paradoxical" calming effect of stimulants seems rarer than tolerance to the euphoric or anorexic effects, it has been reported by Winsberg and associates. ${ }^{8}$ They recommend, when this happens, alternating drugs weekly or allowing a two-day "rest" between five-day treatment periods. True tolerance must be distinguished from a temporary placebo effect or "euphoria cure" followed by deterioration at the same dosage. When this happens at a low dose, an increase will sometimes result in a more permanent improvement with no tolerance. 


\section{Side Effects}

The side effects of stimulants currently appear to be more annoying than dangerous. The common side effects can usually be tolerated when fair warning and assurance have been offered. It may be advisable to preface the mention of side effects with some such remark as "there are some possible side effects which I want to mention so that if they happen, you'll know what they are and not worry about them."

Anorexia is very predictable and should always be mentioned ahead of time. The risk of a child losing his appetite by suggestion is negligible compared to the probability of pharmacologic anorexia. Most authors deem this appetite loss "temporary" and of no consequence for growth. However, Safer, Allen and Barr ${ }^{9}$ have found evidence of mild growth impairment and "rebound" gain in children who stopped medication during summer vacation. This observation underscores the wisdom of withholding stimulants on vacations, weekends, and holidays from a child who has trouble only at school.

Insomnia is usually not much of a problem when the last dose is given no later than early afternoon (morning if it is a time-release capsule). When it does occur, the insomnia may be self-limited or can be handled by readjustment of dosage time or by adding diphenhydramine at bedtime.

Gastrointestinal cramps are probably the most distressing side effects. When they occur, they often lead to loss of patient cooperation. Because only a small proportion of children have them, and because of all the side effects these are the most likely to be induced by suggestion, they should probably not be mentioned in front of the child. However, when opportunity presents, the parents should be told about them as "possible temporary irritations of the stomach until he gets used to the medicine."

The possibility of temporary whininess, sensitivity, or depression should probably be spoken of, but these are not usually a reason for discontinuing medication. The depression, being a "paradoxical" effect, may be a confirmation that the right medication has been found.

Nevertheless, the dosage should be reduced to lighten the depression, at least until the child makes the necessary psychologic adjustments. One boy who threatened suicide with $10 \mathrm{mg}$ of methylphenidate a day adjusted extremely well at $5 \mathrm{mg}$ a day, with dramatic improvement over his predrug state.

Though the literature does not document appreciable cardiovascular disturbances in pooled data at the usual doses, there is anunpublished suspicion that occasional idiosyncratic individuals may suffer such symptoms. Any complaint of headaches, palpitation, or other symptoms ascribable to cardiovascular disturbance should be promptly investigated. Though it is not presently universal practice to monitor pulse and blood pressure, this may be advisable in the future.

Tactile hallucinations (e.g., "skin crawling") or tics warrant a reduction or discontinuance of stimulant therapy. In my experience, these have been rare and do not deserve mention ahead of time.

Sometimes, when a stimulant is effective but rich in side effects, a different stimulant (or a different type of drug) may offer the same benefit with less severe side effects. Sometimes one has to choose between the lesser of two evils, side effects or the original complaint. Such a choice, of course, should be discussed with the patient and his parents.

\section{References}

1. Nichamin, S. J. and Barahal, G. D.: Faulty neurologic integration with perceptual disorders in children. Mich. 
Med. 67: 1071, 1968.

2. Eisenberg, L.: Psychiatric implications of brain damage in children. Psychiatr. Q. 31: 72, 1957.

3. Millichap, J. G.: Drugs in management of hyperkinetic and perceptually handicapped children. JAMA 206: 1527, 1968.

4. Laufer, M. and Denhoff, E.: Hyperkinetic behavior syndrome in children. J. Pediatr. 50: 463, 1967.

5. Fish: The "one child, one drug" myth of stimulants in hyperkinesis. Arch. Gen. Psychiatry 25: 193, 1971.

6. Wender: Minimal Brain Dysfunction in Children. New York, John Wylie and Sons, Inc., 1971.

7. Rosenthal, R. and Jacobson, L.: Pygmalion in the Classroom. New York, Holt, Rinehart, and Winston, Inc., 1968.

8. Winsberg, B. G., Bialer, I., Kupietz, S. and Tobias, J.; Effects of imipramine and dextroamphetamine on behavior of neuropsychiatrically impaired children. Am. J. Psychiatry 128: 1425, 1972.

9. Safer, D., Allen, R, and Barr, E.: Depression of growth in hyperactive children on stimulant drugs. N. Engl. J. Med. 287: 217, 1972. 